\title{
Advances in Quantum Teleportation
}

\author{
S. Pirandola, ${ }^{1}$ J. Eisert,${ }^{2}$ C. Weedbrook,${ }^{3}$ A. Furusawa, ${ }^{4}$ and S. L. Braunstein ${ }^{1}$
}

1 Computer Science and York Centre for Quantum Technologies, University of York, York YO10 5GH, United Kingdom

2 Dahlem Center for Complex Quantum Systems, Freie Universität Berlin, 14195 Berlin, Germany

3 Department of Physics, University of Toronto, Toronto M5S 3G4, Canada

4 Department of Applied Physics, The University of Tokyo, 7-3-1 Hongo, Bunkyo-ku, Tokyo 113-8656, Japan

Quantum teleportation is one of the most important protocols in quantum information. By exploiting the physical resource of entanglement, quantum teleportation serves as a key primitive in a variety of quantum information tasks and represents an important building block for quantum technologies, with a pivotal role in the continuing progress of quantum communication, quantum computing and quantum networks. Here we review the basic theoretical ideas behind quantum teleportation and its variant protocols. We focus on the main experiments, together with the technical advantages and disadvantages associated with the use of the various technologies, from photonic qubits and optical modes to atomic ensembles, trapped atoms, and solid-state systems. Analysing the current state-of-the-art, we finish by discussing open issues, challenges and potential future implementations.

\section{From Science Fiction to Reality}

It has been over two decades since the discovery of quantum teleportation, in what is arguably one of the most interesting and exciting implications of the 'weirdness' of quantum mechanics. Previous to this landmark discovery, this fascinating idea belonged to the realm of science fiction. First coined in a 1931 book by Charles H. Fort ${ }^{1}$, the term teleportation has since been used to refer to the process by which bodies and objects are transferred from one location to another, without actually making the journey along the way. Since then it has become a fixture of pop culture, perhaps best exemplified by Star Trek's celebrated catchphrase "Beam me up, Scotty."

In 1993, a seminal paper ${ }^{2}$ described a quantum information protocol, dubbed quantum teleportation, that shares several of the above features. In this protocol, an unknown quantum state of a physical system is measured and subsequently reconstructed or 'reassembled' at a remote location (the physical constituents of the original system remain at the sending location). This process requires classical communication and excludes superluminal communication. Most importantly, it requires the resource of quantum entanglement ${ }^{3,4}$. Indeed, quantum teleportation can be seen as the protocol in quantum information that most clearly demonstrates the character of quantum entanglement as a resource: Without its presence, such a quantum state transfer would not be possible within the laws of quantum mechanics.

Quantum teleportation plays an active role in the progress of quantum information science ${ }^{5-8}$. On the one hand it is a conceptual protocol crucial in the development of formal quantum information theory, on the other it represents a fun- damental ingredient to the development of many quantum technologies. Schemes such as quantum repeaters ${ }^{9}-$ pivotal for quantum communication over large distances - quantum gate teleportation ${ }^{10}$, measurement-based computing ${ }^{11}$, and port-based teleportation ${ }^{12}$ all derive from the basic scheme. The vision of a quantum network ${ }^{13}$ draws inspiration from it. Teleportation has also been used as a simple tool for exploring 'extreme' physics, such as closed time-like curves ${ }^{14}$ and black-hole evaporation ${ }^{15}$.

Today, quantum teleportation has been achieved in laboratories the world over using many different substrates and technologies, including photonic qubits (e.g., light polarisation $^{16-22}$, time-bin ${ }^{23-25}$, dual rails on chip ${ }^{26}$, spin-orbital qubits $^{27}$ ), nuclear magnetic resonance (NMR) ${ }^{28}$, optical modes $^{29-37}$, atomic ensembles ${ }^{38-41}$, trapped atoms ${ }^{42-46}$, and solid state systems ${ }^{47-50}$. Outstanding performances have been achieved in terms of teleportation distance ${ }^{21,22}$, with satellitebased implementations forthcoming. Attempts at scaling to more complex quantum systems have also been started ${ }^{27}$.

\section{The Basics of Quantum Teleportation}

\section{A. Quantum Teleportation of Qubits and Discrete Variables}

Quantum teleportation was originally described for twolevel quantum systems, so-called qubits ${ }^{2}$. The protocol considers two remote parties, referred to as Alice and Bob, who share two qubits, $A$ and $B$, prepared in a pure entangled state. In the ideal case, this is taken to be maximally entangled, such as $|\Phi\rangle=(|0,0\rangle+|1,1\rangle) / \sqrt{2}$, also known as a Bell pair ${ }^{5}$. At the input, Alice is given another qubit $a$ whose state $\rho$ is 

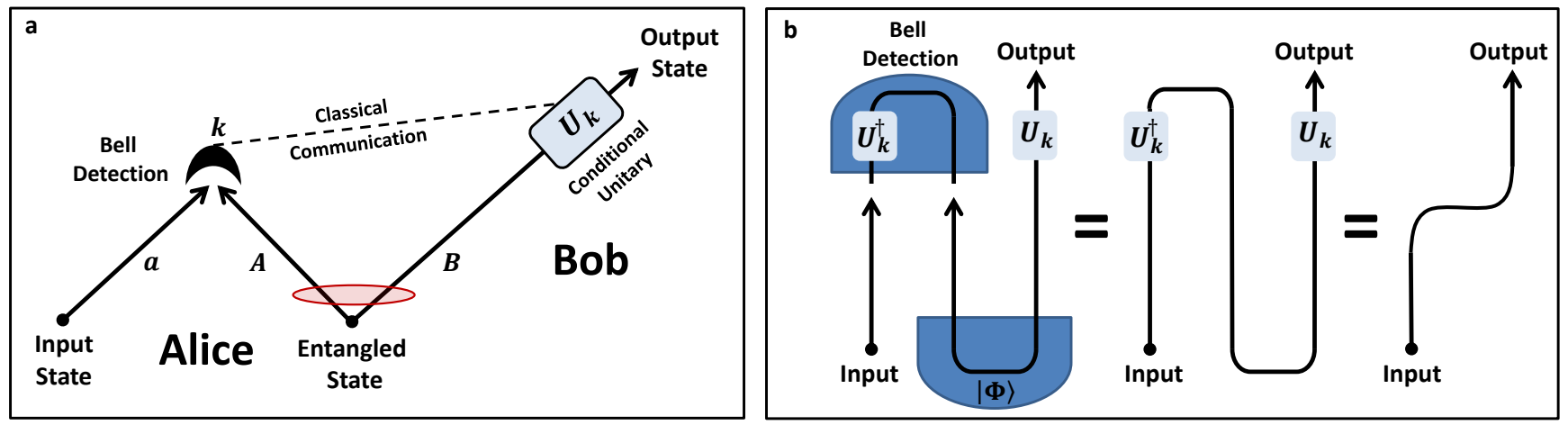

FIG. 1. Panel a shows the basic protocol of quantum teleportation. Alice's unknown input state is teleported to Bob using a shared entangled state and a classical communication channel. Alice performs a Bell state measurement on her systems, $a$ and $A$, and classically communicates the outcome $k$ to Bob. Using the measurement result, Bob applies the conditional unitary $U_{k}$ to his system $B$, retrieving an output state which is an exact replica of Alice's input state in the ideal case $(F=1)$. The outcomes of the Bell detection are: $k=0, \ldots, d^{2}-1$ for DV systems, and $k \in \mathbb{C}$ for continuous-variable $(\mathrm{CV})$ systems. In qubit-teleportation $(d=2)$, the input state is typically pure, the entangled state is a Bell pair, and $U_{k}$ is a qubit Pauli operator $\mathcal{P}_{k}$. In $\mathrm{CV}$ teleportation $(d=\infty)$, the systems are bosonic modes (e.g., optical), the input is typically a coherent state, the entangled state is an EPR state, and $U_{k}$ is a phase-space displacement. Variations on this basic protocol may occur depending on the actual technologies adopted. Panel $\mathbf{b}$ is a representation of the ideal teleportation process in terms of Penrose-inspired space-time diagrams ${ }^{77,78}$. Let us consider a maximally entangled state $|\Phi\rangle$ as a shared quantum resource (i.e., a Bell pair for qubits, or an ideal EPR state for CVs). Then, the Bell detection corresponds to applying $\langle\Phi|\left(U_{k}^{\dagger} \otimes I\right)$ where the outcome $k$ is randomly selected by the measurement. As a result, an arbitrary input state $\rho$ is transformed into $U_{k}^{\dagger} \rho U_{k}$ where the unitary $U_{k}^{\dagger}$ is correspondingly 'undone' by Bob.

unknown. She then performs a joint quantum measurement, called Bell detection ${ }^{51,52}$, which projects her qubits $a$ and $A$ into one of the four Bell states $\left(\mathcal{P}_{k} \otimes I\right)|\Phi\rangle$ with $k=0, \ldots, 3$ where $\mathcal{P}_{k}$ is a qubit Pauli operator ${ }^{5,6}$. As a result, the state of Alice's input qubit has been collapsed by measurement while Bob's qubit $B$ is simultaneously projected onto $\mathcal{P}_{k}^{\dagger} \rho \mathcal{P}_{k}$. In the last (feed-forward) step of the protocol, Alice communicates the classical outcome $k$ of her measurement to Bob, who then applies $\mathcal{P}_{k}$ to recover the original input state $\rho$.

Note that Alice's input state is assumed to be unknown, otherwise the protocol reduces to remote state preparation ${ }^{54}$. Furthermore, this state may itself be part of a larger composite quantum system shared with a third party (in which case, successful teleportation requires reproducing all correlations with that third party). In typical experiments, the input state is been taken to be pure and belonging to a limited alphabet of states, e.g., the six poles of the Bloch sphere ${ }^{5}$. In the presence of decoherence, the quality of the reconstructed state may be quantified by its teleportation fidelity, $F \in[0,1]$. This is the fidelity ${ }^{5}$ between Alice's input state and Bob's output state, averaged over all the outcomes of the Bell detection and input state alphabet. For small values of the fidelity, strategies exist that allow for an imperfect teleportation while making no use of any entangled resource. For example, Alice may directly measure her input state, sending the results to Bob for him to prepare an output state ${ }^{55}$. Such a measure-prepare strategy is called 'classical teleportation' and has the maximum fidelity ${ }^{56}$ $F_{\text {class }}=2 / 3$ for an arbitrary input state or, equivalently, an alphabet of mutually unbiased states, such as the six poles of the Bloch sphere. Thus, one clear benchmark to ensure that quantum resources are utilized is to require fidelity $F>F_{\text {class }}$.

Quantum teleportation is not restricted to qubits, but may involve higher-dimensional quantum systems. For every finite dimension $d$, one can formulate ideal teleportation schemes:
This exploits a basis of maximally entangled state vectors, which can be derived from a given maximally entangled state, and a basis $\left\{U_{k}\right\}$ of unitary operators satisfying ${ }^{57}$ $\operatorname{tr}\left(U_{j}^{\dagger} U_{k}\right)=d \delta_{j, k}$. The scheme follows then the above lines, with the Pauli operators $\mathcal{P}_{k}$ replaced by $U_{k}$ and the classical channel involves distinguishing $d^{2}$ signals. Such a protocol can be constructed for any finite-dimensional Hilbert space, for so-called discrete-variable (DV) systems. See Fig. 1.

\section{B. Quantum Teleportation of Continuous Variables}

Quantum teleportation can also be extended to quantum systems with an infinite-dimensional Hilbert space, known as continuous-variable (CV) systems. These are typically realised by optical bosonic modes, whose electric field can be described by position- and momentum-like quadrature operators $^{7,8}$. Following the first theoretical proposals ${ }^{58,59}$, CV teleportation was demonstrated with optical modes ${ }^{29}$. Other CV systems can be considered, including opto-mechanical systems ${ }^{60,61}$ and collective spins of atomic ensembles ${ }^{38,39,62-64}$.

In the standard $\mathrm{CV}$ protocol with optical modes, the entangled resource corresponds to a two-mode squeezed vacuum state ${ }^{8}$, also known as an Einstein-Podolsky-Rosen (EPR) state. This is a zero-mean Gaussian state ${ }^{7}$ with logarithmic negativity $^{65-67}-\log (\varepsilon)$, where the parameter $\varepsilon \leq 1$ can be computed from its covariance matrix ${ }^{7}$. Suppose Alice is given an input mode $a$ in an unknown state; typically, a coherent state $|\alpha\rangle$ with unknown amplitude $\alpha$. She then applies a CV Bell measurement on her modes, $a$ and $A$, which consists of mixing them on a balanced beam splitter and homodyning the output ports in the conjugate quadratures $\hat{q}_{-}=\left(\hat{q}_{a}-\hat{q}_{A}\right) / \sqrt{2}$ and $\hat{p}_{+}=\left(\hat{p}_{a}+\hat{p}_{A}\right) / \sqrt{2}$. In the last step, Alice communicates the classical outcome $k=q_{-}+i p_{+}$to Bob, who performs a 
conditional displacement on his mode $B$ (see Fig. 1)

The teleportation fidelity for the alphabet of coherent states is given by $F=(1+\varepsilon)^{-1}$. Perfect teleportation occurs only for unbounded entanglement $\varepsilon \rightarrow 0$, where the entangled state approximates the ideal EPR state, realising the perfect correlations $\hat{q}_{A}=\hat{q}_{B}$ and $\hat{p}_{A}=-\hat{p}_{B}$. Without entanglement ( $\varepsilon=1$ ) we have $F=1 / 2$, which is the classical (i.e., no entanglement) threshold for teleporting coherent states ${ }^{68,69}$. A more stringent threshold asks that the teleported state is the best copy of the input allowed by the no-cloning bound ${ }^{70}$, requiring $F>2 / 3$. This basic CV protocol can be extended to more general Gaussian state settings ${ }^{71-76}$.

\section{Variants of Teleportation}

Teleportation is an important primitive which has been extended in various ways. Some of these extensions are actual protocols of quantum technology, while others are of conceptual value in theoretical models. In this section, we mention some of these variants and sketch some recent developments.

\section{A. Entanglement Swapping and Quantum Repeaters}

As previously mentioned, the input to be teleported can itself be part of an entangled state. Teleportation would then also transfer the entanglement. When the Bell detection is performed by a third party, say Charlie acting as the middle relay, this variant is called entanglement swapping. Suppose that Alice and Bob do not share entanglement, but they locally prepare two entangled states; $\rho_{a A}$ at Alice's station and $\rho_{b B}$ at Bob's. They retain systems $a$ and $b$, while sending systems $A$ and $B$ to Charlie for Bell detection. After the measurement outcome $k$ is classically broadcast, Alice and Bob share the conditional output state $\rho_{a b \mid k}$ which is entangled. This can be implemented with DV systems ${ }^{80}$ (e.g., polarisation qubits ${ }^{87-89}$ ), CV systems ${ }^{81-86}$ (e.g., optical modes ${ }^{32,90}$ ), or adopting a hybrid approach involving both DVs and CVs ${ }^{91}$.

This protocol forms the basis for quantum repeaters ${ }^{9}$, in which the combination of entanglement swapping and distilla$\operatorname{tion}^{92,93}$ allows for the distribution of entanglement over large distances. Once that maximal entanglement is distilled along a long chain of repeaters, teleportation between the end-users provides the perfect transfer of quantum information. The remote correlations created by swapping can also be exploited in quantum cryptography: Even when Charlie is present in the role of an eavesdropper (untrusted relay), Alice and Bob can transform their resulting correlations into a secret key ${ }^{94}$.

\section{B. Quantum Teleportation Networks}

Another important extension is to a quantum teleportation network, where $n>2$ parties initially share a multipartite entangled state and teleportation can be performed between any two parties. For simplicity, we describe the simplest case of a 3-party network, where, for example, Alice can teleport to either Bob or Charlie. One simple strategy is assisted teleportation, where Charlie performs a local measurement on his system and broadcasts the result with the aim of improving the fidelity of quantum teleportation from Alice to Bob. This requires Charlie's operation to be tailored so as to increase the bipartite entanglement of the remaining parties.

With qubits, 3-party assisted networks can be constructed using the Greenberger-Horne-Zeilinger state ${ }^{95}$. In particular, this state makes teleportation equivalent to quantum secret sharing ${ }^{96}$, where Alice's quantum information can be recovered by Bob if and only if Charlie assists. With CV systems, 3-party assisted networks can be constructed using Gaussian states $^{71}$, e.g., generated using squeezed vacua at the input of two beamsplitters ${ }^{97}$ as used in the first experimental demonstrations ${ }^{98,99}$. In general, by using one or more squeezed vacua in an interferometer, one can create multi-party assisted networks involving an arbitrary number of bosonic modes ${ }^{8,97}$.

A second strategy is unassisted teleportation, where Charlie rather than helping Alice, also receives a copy of the input. In this case, the protocol corresponds to quantum telecloning, with Alice teleporting to Bob and Charlie simultaneously, though with a teleportation fidelity limited by the nocloning bound (which is $5 / 6$ for qubits ${ }^{100,101}$, and $2 / 3$ for coherent states of CV systems ${ }^{102}$ ). Quantum telecloning, from one sender to two recipients, has been experimentally implemented with polarised photonic qubits ${ }^{103}$ and coherent states of optical modes ${ }^{104}$. Theoretically, the protocol can be formulated for an arbitrary number of recipients, both in the case of qubits $^{105}$ and CV systems ${ }^{106}$.

\section{Quantum Gate Teleportation and Quantum Computing}

Quantum teleportation can be expressed in terms of primitive quantum computational operations ${ }^{107}$ and its protocol can be extended to quantum gate teleportation ${ }^{10,108}$. This idea is rooted in the observation that unitary state manipulation can be achieved by preparing auxiliary entangled states, performing local measurements and applying single-qubit operations. This approach is at the heart of linear-optical quantum computing ${ }^{109}$ and plays an important role in fault-tolerant quantum computation. Basically, one prepares certain gates offline as the entanglement resource for a teleportation protocol ${ }^{5}$. This resource is then also easier to implement fault-tolerantly. Experimentally, two-qubit gates have been teleported ${ }^{110}$.

More generally, such gate teleportation strategies lie at the heart of cluster state quantum computing ${ }^{11}$ and other schemes for measurement-based quantum computing ${ }^{111}$. Here a number of nodes (qubits or qumodes ${ }^{7}$ ) are prepared in a multipartite entangled state (e.g., a cluster state) or another suitable tensor network state. Then, a suitable measurement on one node teleports its state onto another node with the concurrent application of a desired quantum gate ${ }^{11,112}$. Large cluster states can be generated with CV systems ${ }^{113,114}$, with an experiment implemented, involving more than $10^{4}$ optical modes $^{115}$. 


\section{Port-Based Teleportation}

In port-based teleportation ${ }^{12,116}$, Alice and Bob share $n$ Bell pairs, referred to as ports. Alice then performs a suitable joint measurement on the system to be teleported and her $n$ qubits, communicating the outcome $k=1, \ldots, n$ to Bob. Finally, he discards all his systems except qubit $k$, which will be in the unknown state of Alice's input. Compared to conventional teleportation, port-based teleportation has the advantage that Bob does not need to apply a correction at the end of the protocol. The disadvantage is that the teleportation fidelity approaches unity only in the limit of large $n$.

The importance of this scheme is less of a practical nature, due to the large entanglement resources needed (even though this can be improved by entanglement recycling ${ }^{117}$ ), but rather in conceptual studies in quantum information theory. It is an important primitive for devising programmable quantum processors $^{12}$, tailored to store a unitary transformation and then applying it to an arbitrary state. It has also been used in ideas of instantaneous non-local quantum computation ${ }^{118}$, in attacking schemes of position-based cryptography ${ }^{118}$, and in assessments of communication complexity tasks. In fact, advantages in quantum communication complexity have generally been linked to violations of Bell inequalities, with port-based teleportation being the main proof tool ${ }^{119}$.

\section{Experimental Status and Challenges}

We now discuss the main experimental achievements and challenges. Ideally, an experiment of quantum teleportation is successful when the following basic requirements are met:

(1) The input state is arbitrary (within a suitable alphabet).

(2) A third party, say Victor, supplies the input state to Alice and independently verifies Bob's output state (e.g., via quantum state tomography or fidelity estimation).

(3) Alice performs a complete Bell detection, being able to distinguish an entire basis of entangled states.

(4) Teleportation fidelity exceeds the appropriate threshold, achievable by classical measure-prepare strategies.

In the laboratory though, some of these conditions may not be met. In particular, the failure of condition (3) has led to post-selected (or probabilistic) teleportation, where only a subset of the Bell states are accessible. In this case, the protocol has an associated Bell-efficiency, which provides an upper bound to its overall success probability. By contrast, if condition (3) can be met, then one can in principle perform unconditional (or deterministic) teleportation.

The most complete experiments of unconditional teleportation are those with 'real-time' (or 'active') feed-forward, meaning that the outcomes of the Bell measurement are communicated in real-time to Bob, and the conditional unitaries are actively performed on the output state, before being verified by Victor. In other experiments the feed-forward is instead simulated in the post-processing, i.e., the output state is directly measured by Victor and the unitaries are simulated only after the tomographic reconstruction of the state.

\section{A. Photonic Qubits}

The practical realisation of a complete Bell detection is still a major issue for photonic qubits, since the simplest use of linear optics and photodetection allows one to distinguish at most two of the four Bell states ${ }^{51-53}$, thus limiting the Bellefficiency to $50 \%$. In principle, by using linear optics and $n$ ancillary qubits, one can teleport one qubit with better Bellefficiency ${ }^{109}$, approaching $100 \%$ for infinite $n$. This theoretical possibility clearly implies an overhead of quantum resources which poses non-trivial experimental challenges. A similar improvement can be achieved via quantum error correction. One can build an $n$-qubit code correcting $2 n-1$ localised erasures. Encoding the input qubit in such a code would then protect against the inconclusive Bell outcomes, tolerating a probability of erasure approaching $50 \%$ for large $n$. Improving the practical efficiency of the Bell detection with photonic qubits is today an active area of research ${ }^{120-122}$.

The Innsbruck experiment ${ }^{16}$ realised post-selected teleportation of a polarisation qubit with $25 \%$ Bell-efficiency, a performance later improved to $50 \%$ in a $600 \mathrm{~m}$ fibre-optical implementation across the river Danube ${ }^{17}$. A point raised about the Innsbruck experiment was that, since its implementation required Bob's teleported qubit to be detected, condition (2) above was rendered problematic. One might argue that for this experiment teleportation was achieved as a post-diction and was therefore termed "a posteriori teleportation"123.

To achieve complete Bell detection, the Rome experiment ${ }^{18}$ entangled photons in both spatial and polarisation degrees of freedom. The input state was prepared within the setup over one of the entangled photons, therefore losing the ability to teleport entangled or mixed states. This implies that the input state cannot be independently supplied from outside, thus violating condition (2) above. The scheme was later extended to free-space teleportation over $16 \mathrm{~km}$ with real-time feedforward and achieved $89 \%$ fidelity ${ }^{19}$. Complete Bell detection has also been attempted using nonlinear interactions. Unfortunately, this leads to an extremely inefficient protocol. Using optical upconversion ${ }^{20}$, only one out of roughly $10^{10}$ polarisation qubits takes part in the non-linear process.

A photonic qubit has also be realised as a time-bin qubit ${ }^{124}$, i.e., as a single photon populating one of two temporal modes. Time-bin qubits have been teleported along a $2 \mathrm{~km}$ telecom fibre with $25 \%$ Bell-efficiency and $\simeq 81 \%$ fidelity $^{23}$. The qubit was teleported between photons of different wavelengths (from $1.3 \mu \mathrm{m}$ to $1.55 \mu \mathrm{m}$ ). This experiment was extended to a relay configuration ${ }^{24}$, where the Bell detection was performed by a third party, connected with Alice's input state and the entangled source by means of $2 \mathrm{~km}$ fibres. Such a setup has the challenge to preserve the indistinguishability of the photons involved in the Bell detection. See ref. 25 for an implementation on a commercial telecom network.

Photonic qubits are an excellent substrate for achieving long distances. This is particularly true in free space where 

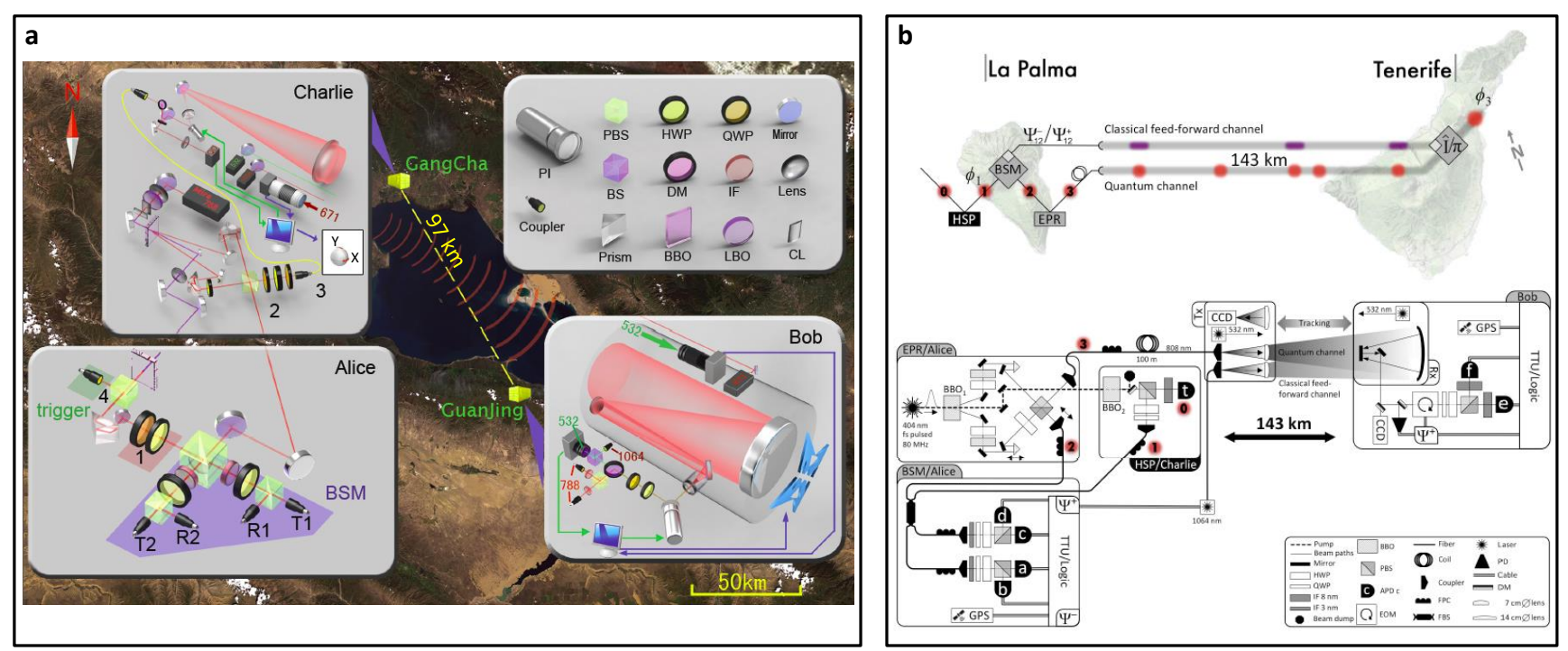

FIG. 2. Long-distance quantum teleportation with polarisation qubits. The experiment ${ }^{21}$ in panel a considered a free-space link of $\simeq 100 \mathrm{~km}$ at ground level across the Qinghai lake in China. Teleportation was implemented with $50 \%$ Bell-efficiency and a fidelity $\gtrsim 80 \%$. The channel attenuation between Alice and Bob was variable between 35 and $53 \mathrm{~dB}$. Besides atmospheric loss, a major attenuation came from the beam spreading wider than the $40 \mathrm{~cm}$ aperture of the receiver telescope. The experiment relied on a point-and-track control system for the telescopes to compensate atmospheric turbulence and ground settlement. Entanglement distribution over two $\simeq 50 \mathrm{~km}$ links (for a total of $\simeq 80 \mathrm{~dB}$ loss) was also implemented. The experiment ${ }^{22}$ in panel $\mathbf{b}$ considered a free-space link of $143 \mathrm{~km}$ at 2,400 m altitude between the Canary islands of La Palma and Tenerife. Teleportation was implemented with $50 \%$ Bell-efficiency (with active feed-forward) and a fidelity $\gtrsim 83 \%$. A telescope with $1 \mathrm{~m}$ aperture was used at Bob's site, and the total channel attenuation varied between 28 and $39 \mathrm{~dB}$. Besides a tracking system, the experiment involved techniques to enhance the signal-to-noise ratio, including low-noise single-photon detectors with large active areas and entanglement-assisted clock synchronisation. Figures adapted with permission from: a, ref. 21, (c) 2012 NPG; b, ref. 22 , (C) 2012 NPG.

birefringence is weak and photon absorption is small at optical frequencies (e.g., compared to fibres). Two recent experiments with polarisation qubits in free space ${ }^{21,22}$ achieved very long distances, suggesting that the atmosphere can be traversed, and ground-to-satellite implementations are within reach of current technology. See Fig. 2 for more details.

A complementary challenge is the photonic miniaturisation of teleportation for applications in quantum processors based on linear optics and probabilistic gates ${ }^{109}$. Ref. 26 teleported a dual-rail qubit (a single photon encoded in one of two spatially-separated modes) within a configurable photonic chip. Bell detection was performed with an efficiency of $1 / 27$, and a fidelity of $89 \%$ was extrapolated by simulating the feed-forward operations in the post-processing, after the tomographic reconstruction of Bob's output. Active feedforward remains a major challenge on photonic chips due to the need for ultrafast photon detection and integrated electronics operating with terahertz bandwidths.

Finally, we have witnessed the teleportation of multiple degrees of freedom of a composite quantum system. Ref. 27 simultaneously teleported the spin (polarisation) and orbital angular momentum of a single photon, with a fidelity ranging from $57 \%$ to $68 \%$. This is above the classical threshold of $40 \%$ given by the optimal state estimation on a single copy of a two-qubit system. Besides a hyper-entangled source, the experiment exploited a hyper-entangled Bell detection with efficiency of $1 / 32$. To measure the two degrees of freedom, the Bell detection was designed as a quantum non-demolition measurement involving an additional teleportation setup.

\section{B. Nuclear Magnetic Resonance}

Complete Bell detection is achievable in NMR teleportation, where the qubits are nuclear spins. Ref. 28 considered the spins of two Carbon nuclei $\left(\mathrm{C}_{1}\right.$ and $\left.\mathrm{C}_{2}\right)$ and that of a Hydrogen nucleus $(\mathrm{H})$ in a molecule of labelled trichloroethylene (more precisely, an ensemble of such molecules, so that the results are intended to be averaged). After entanglement between $\mathrm{C}_{1}$ and $\mathrm{H}$ was created by spin-spin interactions, teleportation was realised from $\mathrm{C}_{2}$ to $\mathrm{H}$, by making a complete Bell detection on the two Carbon nuclei ${ }^{28}$. This detection was performed in two steps ${ }^{107}$ : The Bell basis was first rotated to the computational basis by radio-frequency (RF) pulses and a spin-spin coupling; then, a projection on this basis was done by exploiting the natural phase decoherence of the Carbon nuclei (the fastest in the molecule, with times $\leq 0.4 \mathrm{~s}$ ). Since NMR teleportation is limited to inter-atomic distances, it is not suitable for quantum communications, but could still be used as a subroutine for NMR quantum computing.

\section{Optical Modes}

The first and simplest resolution of the Bell detection problem came from the use of CV systems, in particular, optical 

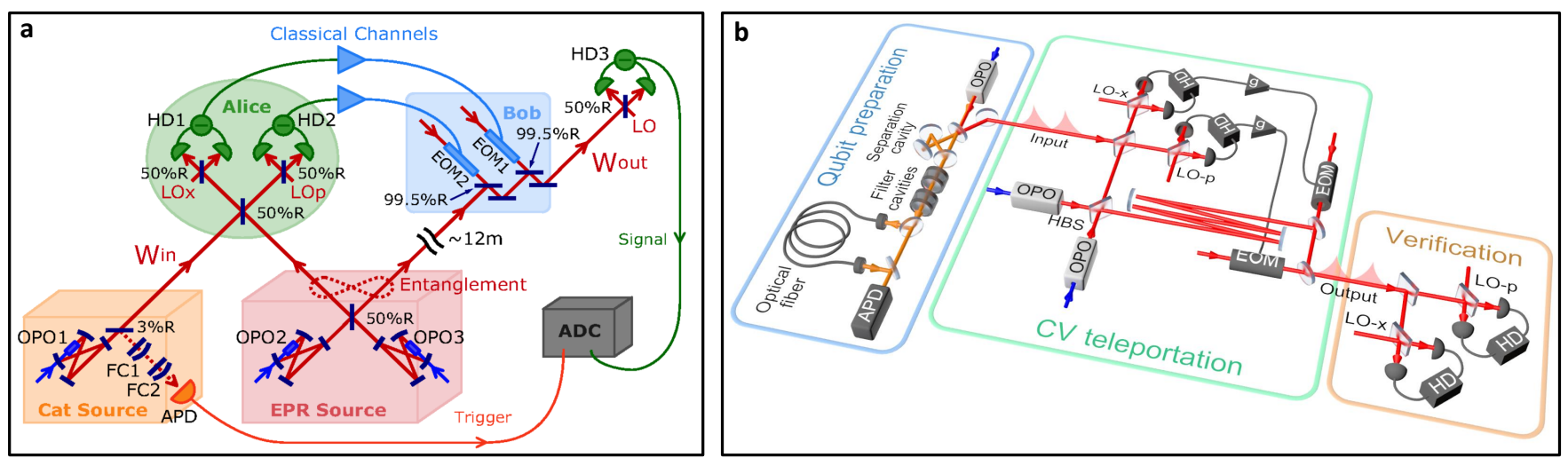

FIG. 3. Quantum teleportation with optical modes. Panel a is the setup of a full CV teleportation experiment with optical modes ${ }^{35}$. Here an EPR state is generated by using two optical parametric oscillators (OPOs) and a balanced beamsplitter. At Alice's station, part of the EPR state is combined with the input state (here a cat state) in a CV Bell detection, i.e., another balanced beamsplitter with two homodyne detectors at the output ports. The classical outcomes are transmitted to Bob who displaces his part of the EPR state by using two EOMs. Note that both the preparation of the input state and the verification of the output state are independently done by Victor. Panel b shows a hybrid teleportation experiment where a time-bin qubit (prepared on the left) is teleported via CV teleportation (the middle setup), involving an EPR state, a complete CV Bell detection and EOMs ${ }^{37}$. Figures adapted with permission from: a, ref. 35, (c) 2011 AAAS; b, ref. 37 , (C) 2013 NPG.

modes $^{29}$. As previously mentioned, the $\mathrm{CV}$ version of this measurement can easily be implemented with linear optics, using a balanced beamsplitter followed by two conjugate homodyne detections at the output ports. Both the beamsplitter visibility and the homodyne quantum efficiency can be extremely high, so that the overall detection can approach $100 \%$ efficiency. CV teleportation satisfies all the conditions (1)(4) stated above, with the only restriction that the input states must belong to a specific alphabet, as a consequence of the infinite-dimensionality of the Hilbert space. The first experiment with optical modes ${ }^{29}$ considered an alphabet of fixedenergy phase-modulated coherent states, achieving a fidelity of $\simeq 58 \%$, above the relevant classical threshold.

This experiment has later been improved to beat the nocloning bound, reaching a fidelity of $\simeq 70 \%$ in ref. 32 and $\simeq 76 \%$ in ref. 33 . These high-fidelity $\mathrm{CV}$ teleporters are able to transfer nonclassical features such as non-positive Wigner functions. Other experiments with coherent states involved phase scanning at different amplitudes (ref. 31 with fidelity $\simeq 61 \%$ ) and simultaneous amplitude and phase modulation (ref. 30 with fidelity $\simeq 64 \%$ ). Besides coherent states, other classes of states have been teleported, including squeezed states $^{33,34}$, Schrödinger cat states ${ }^{35}$ and entangled states (see entanglement swapping ${ }^{32,90,91}$ ). For typical setups see Fig. 3.

Unfortunately, CV teleportation fidelities cannot reach $100 \%$, since CV systems do not allow a maximally entangled state to be prepared with finite resources. Realistic EPR states, with good but finite squeezing, can be realised by producing two single-mode squeezed states via parametric downconversion (e.g., in optical parametric oscillators) and combining them on a balanced beamsplitter. Even with the high levels of squeezing currently feasible $(\simeq 10 \mathrm{~dB})$, this resource also requires good phase locking in a teleportation experiment. The current record for CVs is a fidelity of $\simeq 83 \%^{36}$.

In order to overcome the limitations due to finite squeezing, CV teleportation has been 'integrated' with DVs into a hybrid formulation ${ }^{125,126}$. Ref. 37 realised the unconditional
CV quantum teleportation of a photonic qubit, by applying a broadband CV teleporter to the temporal modes of a timebin qubit. Such an approach aims to simultaneously solve the problem of a complete Bell detection (thanks to CVs) as well as achieving a high teleportation fidelity thanks to DVs. Using just moderate levels of squeezing, one can achieve fidelities $\gtrsim 80 \%$. See also Fig. 3(b).

Optical modes are well suited for integration into communication technologies, thanks to the use of high-performance homodyne detectors and off-the-shelf parts, like the electrooptical modulators (EOMs) used for the input preparation and the output displacements. While it is unclear whether the $\mathrm{CV}$ teleportation distance is limited only to middle-range distances due to an increased fragility with respect to loss (all previous experiments have been table-top), the possibility to go broadband and achieve high-rates is clearly appealing.

\section{Atomic Ensembles}

Quantum teleportation may be performed between quantum systems of a different nature, for example, between light and matter. Ref. 38 was the first experiment to show this feature by teleporting coherent states of an optical mode onto the collective spin of an atomic ensemble, composed of around $10^{12}$ room temperature Caesium atoms. Since the collective transverse atomic spin components can be described by quadrature operators, this scheme implements a CV teleportation scheme.

Light-matter entanglement was generated by sending a strong light pulse through Bob's atomic sample ${ }^{38}$, yielding coherent scattering with the light being subject to the Faraday effect and the atoms evolving by the dynamic Stark effect. The optical output was combined with another optical pulse (modulated by an EOM) at Alice's station, where a complete Bell measurement was performed using a balanced beamsplitter followed by two sets of polarisation homodyne detectors. Conditioned on the outcome, Bob performed spin rotations on 

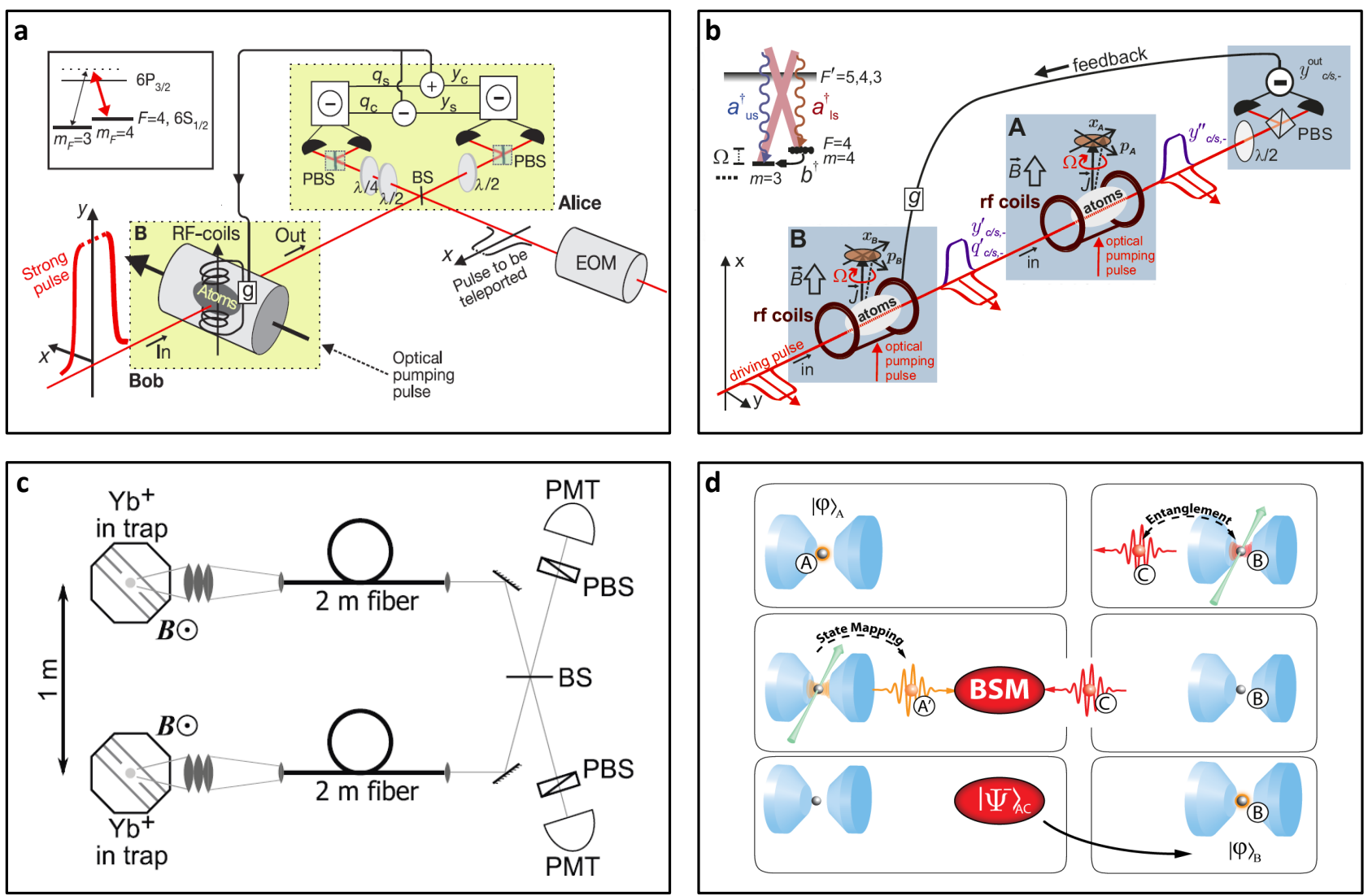

FIG. 4. Quantum teleportation with matter. Upper panels (a-b) show teleportation experiments with atomic ensembles of Caesium atoms at room temperature. Panel a shows the light-to-matter teleportation of a coherent state of an optical mode into a collective atomic spin ${ }^{38}$, while panel $\mathbf{b}$ shows the matter-to-matter teleportation between the collective spins of two atomic ensembles ${ }^{39}$ (see text for more details). Lower panels (c-d) show setups with single trapped atoms, where teleportation is achieved by an incomplete Bell detection on emitted photonic qubits. Panel $\mathbf{c}$ shows the teleportation between trapped Ytterbium ions, confined in independent Paul traps ${ }^{45}$, separated by $1 \mathrm{~m}$. Each atomic qubit was encoded in the hyperfine states of the ${ }^{2} S_{1 / 2}$ level having coherence times of $\simeq 2.5 \mathrm{~s}$, and were manipulated by resonant microwave pulses at $\simeq 12.6 \mathrm{GHz}$. Spontaneously emitted photons were coupled into fibres, and directed to a beamsplitter, where the output ports were filtered in polarisation and detected by single-photon PMTs (implementing a Bell detection with $25 \%$ efficiency). Panel d shows the steps of teleportation between two neutral Rubidium atoms trapped in optical cavities separated by a $21 \mathrm{~m}$ optical fibre ${ }^{46}$. Qubits were encoded in two Zeeman states of the atomic ground-state manifold. Atom-photon entanglement and state mapping were efficiently achieved by vacuum-stimulated Raman adiabatic passage. The generated photonic qubits were then subject to an incomplete Bell detection ( $25 \%$ efficiency). Figures adapted with permission from: a, ref. 38, (c) 2006 NPG; b, ref. 39, (c) 2013 NPG; c, ref. 45, (c) 2009 AAAS; d, ref. 46, (c) 2013 APS.

the atoms applying RF magnetic field pulses. See Fig. 4(a).

In this way, unconditional light-to-matter teleportation was achieved $^{38}$ with a fidelity $\gtrsim 58 \%$. All the conditions (1)-(4) stated above were achieved, for an input alphabet of coherent states. We note that atomic ensembles have the potential for good quantum memories ${ }^{128}$ with sub-second coherence times and millisecond storage times ${ }^{131}$. This is an important feature for the construction of a scalable quantum network where teleportation is used to store flying data (optical modes) into stationary media (atomic ensembles).

This experiment was extended to unconditional matter-tomatter CV teleportation ${ }^{39}$, between two ensembles of around $10^{12}$ room temperature Caesium atoms, by using a four-wave mixing interaction. As before, Bob's ensemble (B) was subject to a strong driving pulse whose scattering created a copropagating sideband field $(\mathrm{C})$ entangled with its collective spin. This field reached Alice's ensemble (A) prepared in a coherent state by RF magnetic field pulses. The interaction with A led to a partial mapping of its state onto $\mathrm{C}$, which was then subject to polarisation homodyning. Conditioned on the outcome, Bob rotated the spin of B via RF magnetic field pulses completing the teleportation from A to B with a fidelity $\gtrsim 55 \%$. This deterministic protocol was also used to teleport time-evolving spin states, thus realizing the first example of stroboscopic teleportation. See Fig. 4(b).

Light-to-matter teleportation has also been achieved with DVs by using cold atomic ensembles ${ }^{40}$. This increased the distance and fidelity but made the protocol probabilistic due to the $50 \%$ Bell efficiency. Ref. 40 teleported a polarisation qubit over $7 \mathrm{~m}$ in fibre onto a collective atomic qubit, made from two cold ensembles of $\simeq 10^{6}$ Rubidium atoms. Teleportation was performed with $\simeq 78 \%$ fidelity, and the output state was stored for $\simeq 8 \mu \mathrm{s}$. This protocol was extended to matterto-matter teleportation, between two cold ensembles of $\simeq 10^{8}$ 
Rubidium atoms (at $\simeq 100 \mu \mathrm{K}$ ) connected by a $150 \mathrm{~m}$ optical fibre ${ }^{41}$. A single collective atomic excitation (spin wave) was teleported between the ensembles with the aid of polarisation qubits. By Raman scattering, Bob's spin wave qubit (B) was entangled with a photonic qubit (B') travelling to Alice. Here, Alice's spin wave qubit (A) was converted into another photonic qubit (A'). Finally, the Bell detection of A' and B' teleported the state of A onto B. A fidelity of $\simeq 88 \%$ with storage times of $\simeq 129 \mu$ s was reported. Note that sub-second storage times are possible with cold atomic ensembles ${ }^{132}$.

\section{E. Trapped Atomic Qubits}

Unconditional qubit teleportation can be realised with trapped ions ${ }^{42-44}$, with all conditions (1)-(4) being satisfied. Even if the distances are very limited, due to the short-range Coulomb interactions, the storage times in these systems are very long. This makes them good quantum memories for using teleportation as a subroutine in quantum computing.

Ref. 42 considered three Beryllium ions $\left({ }^{9} \mathrm{Be}^{+}\right)$confined in a segmented linear Paul trap, with an inter-ion spacing of $3 \mu \mathrm{m}$. Qubits were provided by ground-state hyperfine levels, coupled via stimulated Raman transitions. Resonance fluorescence was used for complete Bell detection and, exploiting spin-echo pulses, a fidelity of $\simeq 78 \%$ was achieved. Independently, ref. 43 considered three Calcium ions $\left({ }^{40} \mathrm{Ca}^{+}\right)$in a linear Paul trap, with an inter-ion distance of $5 \mu \mathrm{m}$. An ion qubit was encoded in a superposition of the $S_{1 / 2}$ ground state and the metastable $D_{5 / 2}$ state (with lifetime $\simeq 1.16 \mathrm{~s}$ ) and manipulated by laser pulses tuned to the $729 \mathrm{~nm}$ quadrupole transition. Complete Bell detection was performed by observing resonance fluorescence on photo-multiplier tubes (PMTs). Using re-phasing spin-echo pulses, a fidelity of $\simeq 75 \%$ was reported with storage lifetimes of $\simeq 10 \mathrm{~ms}$. This second experiment was improved ${ }^{44}$ to achieve a fidelity of $\simeq 83 \%$.

To increase the distance beyond micrometers, one could use a photonic-matter interface where photonic qubits are employed as quantum carriers for state mapping and entanglement distribution, though a practical linear-optics implementation would provide incomplete Bell detection. This approach was followed by ref. 45 , which reported the probabilistic teleportation between two Ytterbium ions $\left({ }^{171} \mathrm{Yb}^{+}\right)$with $\simeq 90 \%$ fidelity (see Fig. 4(c) for other details). This setup provides long coherence times but the free-space interface suffers from inefficient photon collection. An alternate solution may come from cavity quantum electrodynamics, where collection efficiencies are enhanced by the strong atom-cavity coupling, but at the expense of shorter coherence times. Following this idea, ref. 46 reported the probabilistic teleportation between two neutral Rubidium atoms $\left({ }^{87} \mathrm{Rb}\right)$ trapped in two distant optical cavities, with a fidelity $\simeq 88 \%$. See Fig. 4(d) for details.

\section{F. Solid State Systems}

Probabilistic light-to-matter teleportation can be performed between photonic qubits and solid-state quantum memories, composed of semiconductor quantum dots $(\mathrm{QDs})^{47}$ or rareearth doped crystals ${ }^{48}$. The first experiment ${ }^{47}$ probabilistically teleported a photonic frequency qubit generated by a neutral QD (i.e., a single-photon pulse in a superposition of two frequencies) onto the electron spin of a charged QD separated by $5 \mathrm{~m}$ in a different cryostat. A fidelity of $\simeq 78 \%$ was reported for the four equatorial poles of the Bloch sphere (for which $F_{\text {class }}=3 / 4$ ) with a spin coherence time extended to $\simeq 13 \mathrm{~ns}$ using spin-echo pulses (see Fig. 5(a) for details).

The second experiment ${ }^{48}$ probabilistically teleported a telecom-wavelength polarisation qubit onto a single collective excitation of a rare-earth doped crystal (a neodymium-based quantum memory that stored photons for $50 \mathrm{~ns}$ ) achieving a fidelity of $\simeq 89 \%$. One state was teleported over long distance with slightly reduced fidelity, considering a relay configuration where the Bell detection was performed at the output of two $12.4 \mathrm{~km}$ long fibres (see Fig. 5(b) for details). This approach is promising for the availability of quantum memories with long coherence times ${ }^{133}$.

Unconditional matter-to-matter teleportation has also been achieved on solid state devices. Ref. 49 considered superconducting transmon qubits separated by $6 \mathrm{~mm}$ and coupled to waveguide resonators in a circuit quantum electrodynamics setup, as shown in Fig. 5(c). The experiment realised postselected teleportation (fidelity $\simeq 81 \%$ ), unconditional teleportation (fidelity $\simeq 77 \%$ ), also including real-time feed-forward (fidelity $\simeq 69 \%$ ) at a rate of $10^{4} \mathrm{~Hz}$ with microsecond coherence times. The setup employed a quantum bus technology scalable to more complex planar architectures, with nontrivial implications for solid-state quantum computing.

Finally, ref. 50 teleported between two nitrogen-vacancy centres in diamonds separated by $3 \mathrm{~m}$, with complete Bell detection and real-time feed-forward. These centres were addressed by cryogenic confocal microscopes, and their spins manipulated by spin-resolved optical excitations and microwave pulses. The electronic spins of the centres were entangled via entanglement swapping: coupling them with optical fields which were subsequently overlapped at a beamsplitter and finally detected. Upon success of this procedure, the nitrogen nuclear spin of the first centre was prepared and Belldetected together with the electronic spin of the same centre. Teleportation onto the electronic spin of the second centre was achieved with an estimated fidelity of $\simeq 86 \%$. See Fig. 5(d). Nitrogen-vacancy centres are particularly attractive for combining an optical interface (via the electron spin) with a longlived nuclear spin memory, having coherence times exceeding the second at room temperature ${ }^{134}$.

\section{Discussion and Outlook}

We summarise the optimal experimental performances achievable by various substrates and techniques in the table below. It is clear that there is not yet an ideal quantum system or technology which is able to excel in all parameters. Still, we may try to identify preferred candidates in relation to specific applications of quantum teleportation. Suitable hybridization of these candidates with compatible substrates and 

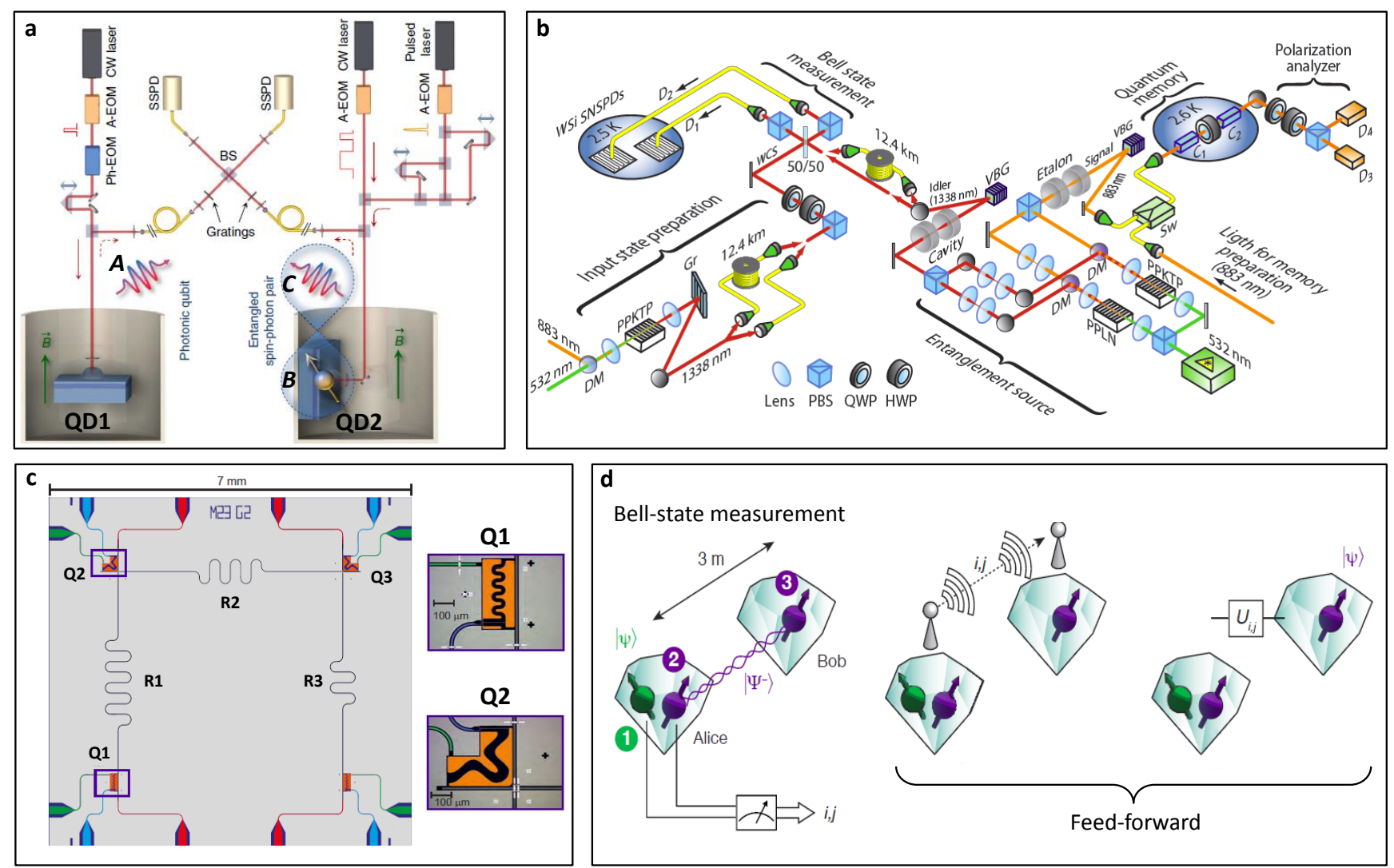

FIG. 5. Quantum teleportation with solid state systems. The setup in panel a shows the teleportation of a photonic frequency qubit $A$, generated by exciting a neutral quantum dot (QD1 in the figure), onto the electron spin $B$ of a charged quantum dot (QD2) ${ }^{47}$. By means of resonant optical excitation, the spin $B$ was entangled with a second frequency qubit $C$, sent to interfere with $A$ in a Hong-Ou-Mandel interferometer. A coincidence detection at the output of the interferometer heralded a successful photonic-to-spin teleportation with $25 \%$ Bell efficiency. Panel b shows the setup for teleporting a telecom polarisation qubit onto a rare-earth doped crystal quantum memory ${ }^{48}$. Two polarisation-entangled photons were generated via spontaneous parametric downconversion in nonlinear waveguides, and one of the photons (at $883 \mathrm{~nm}$ ) was stored in the quantum memory $10 \mathrm{~m}$ away. The other photon (at $1338 \mathrm{~nm}$ ) was sent to interfere with the input polarised photon at the Bell analyzer ( $25 \%$ Bell efficiency), where they were measured by superconducting-nanowire single-photon detectors operating at $2.5 \mathrm{~K}$. Teleportation of the specific state $|+\rangle$ was extended to a total of $\simeq 24.8 \mathrm{~km}$ in fibre. Panel $\mathbf{c}$ shows the chip design for teleportation between two superconducting transmon qubits, from Q1 to Q3, using the middle qubit Q2 and the two resonators R1 and R2, which act as quantum buses for entanglement generation and complete Bell detection ${ }^{49}$. The qubits were manipulated by applying microwave pulses through individual charge gate lines, and Bell state detection was performed with Josephson parametric amplifiers. Panel $\mathbf{d}$ shows the steps of teleportation between two nitrogenvacancy centres in diamonds ${ }^{50}$. Once the electronic spins ( 2 and 3, in the figure) are entangled, the nitrogen nuclear spin (1) is Bell-detected together with the electronic spin (2) of the same centre. Finally, real-time feed-forward yields teleportation onto the other electron spin (3). Figures adapted with permission from: a, ref. 47, (c) 2013 NPG; b, ref. 48, (c) 2014 NPG; c, ref. 49, (c) 2013 NPG; d, ref. 50 , (c) 2014 AAAS.

techniques may provide the most promising future developments for quantum teleportation and its applications.

Short-distance teleportation $(\lesssim 1 \mathrm{~m})$ as a quantum computing subroutine is promising on solid-state devices, with the best approach being circuit $\mathrm{QED}^{142}$. In particular, superconducting transmon qubits may guarantee both deterministic and high-fidelity teleportation on chip ${ }^{49}$. They also allow the implementation of real-time feed-forward, which appears to be challenging with photonic chips ${ }^{26}$. Moreover, they provide a more scalable architecture and better integration to existing technology compared to earlier approaches such as trapped-ions ${ }^{42-44}$. Currently the only downside with these systems appears to be their limited coherence time $(\lesssim 100 \mu$ s). This problem can be overcome by integrating circuit QED with solid-state spin-ensemble quantum memories (nitrogen- vacancy centres or rare-earth doped crystals) which can provide very long coherence times for quantum data storage. This is currently one of the biggest efforts of the community ${ }^{139,140}$.

Teleportation-empowered quantum communication at the metropolitan scale (a few kms), could be developed using optical modes ${ }^{29,36}$. For sufficiently low loss, these systems provide high rates and bandwidths. Experiments may be extended from table-top to medium-range implementations in fibre or free-space and the potential integration with ensemblebased quantum memories. Longer distances but lower rates may be achieved via a hybrid approach ${ }^{37}$ or by developing good quantum repeaters based on non-Gaussian operations ${ }^{93}$.

Long-distance quantum teleportation (beyond $100 \mathrm{~km}$ ) is an active area but still affected by an open problem. Polarisation qubits are the best carriers for (low-rate) teleportation 


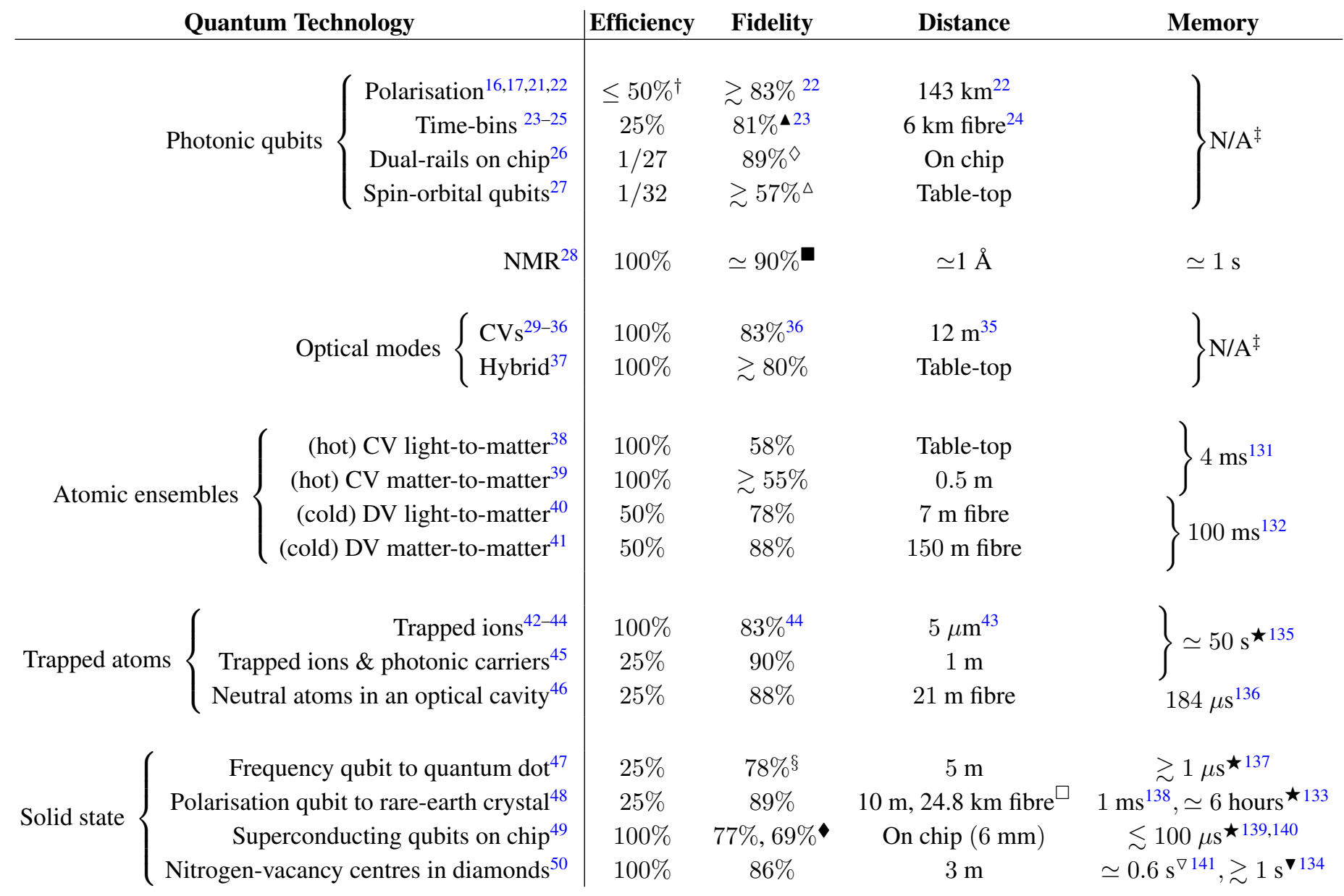

TABLE I. Comparison between quantum teleportation technologies in terms of various quality factors, including the practical efficiency of the Bell state analyzer, the maximum teleportation fidelity, the maximum distance of teleportation in free-space (or fibre, where explicitly indicated), and the storage time of associated quantum memories. The storage time can be either the typical decay time of the stored qubit (estimated from the retrieval efficiency of the memory) or the time over which the write-read fidelity drops below the classical threshold value (e.g., this is the definition used for CVs). Coherence time is shown in some cases. Notes: $\dagger$ Excluding setups with internal qubit preparation ${ }^{18,19}$ for which Bell-efficiency is $100 \%$; $\ddagger$ Full optical delays based on fibres or cavities have very short storage times; $\boldsymbol{\Delta}$ A fidelity of $93 \%$ was achieved for the four equatorial states ${ }^{25} ; \diamond$ Extrapolated fidelity with feed-forward simulated in the post-processing; $\triangle$ Two-qubit teleportation, whose corresponding classical fidelity is $40 \%$; Entanglement fidelity ${ }^{28}$; $\star$ Coherence time for this type of quantum memory; $\S$ Equatorial states teleported; $\square$ One state teleported over $24.8 \mathrm{~km}$ in fibre with a fidelity of $81 \%$; Including real-time feed-forward; $\nabla$ Coherence time for electronic spins at low temperatures $(\simeq 77 \mathrm{~K}) ; \nabla$ Coherence time for nuclear spins at room temperature.

over long fibres and free-space links, but currently make the protocol probabilistic due to the incomplete Bell detection. While probabilistic teleportation and entanglement swapping are acceptable for tasks such as entanglement distillation and quantum cryptography, the situation is clearly different for quantum communication where the input quantum information must be fully preserved.

Accepting this probabilistic nature, satellite-based implementations are within the reach of current technology. Besides the integration of tracking techniques, the main challenge comes from the high loss induced by beam spreading. This may be overcome in a configuration where entanglement is distributed from satellite to large-aperture ground-station telescopes. Assuming a $20 \mathrm{~cm}$ aperture satellite at $\simeq 600 \mathrm{~km}$ altitude and $1 \mathrm{~m}$ aperture telescopes on the ground, one can estimate about $75 \mathrm{~dB}$ loss for a two-downlink channel, which is less than the $\simeq 80 \mathrm{~dB}$ loss achieved at ground level for entanglement distribution ${ }^{21}$. Ground-to-satellite or inter-satellite implementations are more challenging.

The future use of quantum teleportation as a building block of a scalable quantum network strictly depends on its integration with quantum memories. Such memories must come with excellent radiation-matter interfaces, in terms of conversion efficiency, write-read fidelity, storage time and bandwidth (high rate and storage capacity) ${ }^{127-130}$. In the first place, this would allow us to use quantum repeaters for extending quantum communication well beyond direct transmission by way of quantum error correcting codes. The development of good 
quantum memories would enable not only the distribution of entanglement across a network and quantum communication via teleportation, but also the ability to coherently process the stored quantum information. This could ultimately transform the network into a world-wide distributed quantum computer or backbone for a future quantum internet ${ }^{13}$.

From this point of view, atomic ensembles are traditionally considered appealing for their efficient light-to-matter conver$\operatorname{sion}^{128,129}$ and their millisecond storage times, which can approach the $\simeq 100 \mathrm{~ms}$ required by light for transmission on a global scale. However, more promising developments are today coming from solid state systems, where excellent spinensemble quantum memories offer direct integration with the scalable architecture of circuit QED. These memories may not only extend the coherence time of circuit QED but also provide an optical-microwave interface for inter-converting propagating optical/telecom photons with on chip microwave photons ${ }^{140,143}$. Thus, a revised hybrid architecture for the future
Quantum Internet will likely be based on long-distance quantum optical communication suitably interfaced with solidstate nodes for quantum information processing.

\section{Acknowledgements}

S. P. has been supported by the Leverhulme Trust (qBIO fellowship) and the EPSRC, via qDATA (Grant No. EP/L011298/1) and the UK Quantum Communications Hub (Grant No. EP/M013472/1). J. E. has been supported by BMBF (Q.com), the EU (SIQS), and the ERC (TAQ). The authors would like to acknowledge useful feedback from U. L. Andersen, G. Chiribella, N. Gisin, A. Imamoglu, C.-Y. Lu, P. van Loock, S. Mancini, C. Monroe, S. Olmschenk, J. W. Pan, W. Pfaff, E. Polzik, S. Popescu, T. C. Ralph, V. Scarani, C. Simon, R. Thew, W. Tittel, A. Wallraff, and D. J. Wineland.
${ }^{1}$ Fort, C. H. Lo! (Claude Kendall, Publisher, New York, 1931).

2 Bennett, C. H., Brassard, G., Crepeau, C., Jozsa, R., Peres, A. \& Wootters, W. K. Teleporting an unknown quantum state via dual classical and Einstein-Podolsky-Rosen channels. Phys. Rev. Lett. 70, 1895 (1993).

3 Horodecki, R., Horodecki, P., Horodecki, M. \& Horodecki, K. Quantum entanglement. Rev. Mod. Phys. 81, 865 (2009).

4 Eisert J. \& Plenio, M. B. Introduction to the basics of entanglement theory in continuous-variable systems, Int. J. Quant. Inf. 1, 479 (2003).

5 Nielsen, M. A. \& Chuang, I. L. Quantum computation and quantum information (Cambridge University Press, Cambridge, 2000).

${ }^{6}$ Wilde, M. M. Quantum information theory (Cambridge University Press, Cambridge, 2013).

7 Weedbrook, C., et al. Gaussian Quantum Information. Rev. Mod. Phys. 84, 621 (2012).

8 Braunstein, S. L. \& van Loock, P. Quantum information theory with continuous variables. Rev. Mod. Phys. 77, 513 (2005).

9 Briegel, H.-J., Dür, W., Cirac, J. I. \& Zoller, P. Quantum repeaters: The role of imperfect local operations in quantum communication. Phys. Rev. Lett. 81, 5932 (1998).

${ }^{10}$ Gottesman, D. \& Chuang, I. L. Demonstrating the viability of universal quantum computation using teleportation and singlequbit operations. Nature 402, 390-393 (1999).

11 Raussendorf, R. \& Briegel, H. J. A one-way quantum computer. Phys. Rev. Lett. 86, 5188 (2001).

12 Ishizaka, S. \& Hiroshima, T. Asymptotic teleportation scheme as a universal programmable quantum processor. Phys. Rev. Lett. 101, 240501 (2008).

13 Kimble, H. J. The quantum internet. Nature 453, 1023 (2008).

14 Lloyd, S. et al. Closed timelike curves via post-selection: theory and experimental demonstration. Phys. Rev. Lett. 106, 040403 (2011).

15 Lloyd, S. \& Preskill, J. Unitarity of black hole evaporation in final-state projection models. JHEP 08, 126 (2014).

${ }^{16}$ Bouwmeester, D., Pan, J. W., Mattle, K., Eibl, M., Weinfurter, H. $\&$ Zeilinger, A. Experimental quantum teleportation. Nature 390, 575 (1997).
17 Ursin, R., et al. Quantum teleportation across the Danube. Nature 430, 849-849 (2004).

18 Boschi, D., Branca, S., De Martini, F., Hardy, L. \& Popescu, S. Experimental realisation of teleporting an unknown pure quantum state via dual classical and Einstein-Podolski-Rosen channels. Phys. Rev. Lett. 80, 1121 (1998).

19 Jin, X.-M., et al. Experimental free-space quantum teleportation. Nature Photonics 4, 376 (2010).

${ }^{20}$ Kim, Y.-H., Kulik, S. P. \& Shih, Y. Quantum teleportation of a polarisation state with complete Bell state measurement. Phys. Rev. Lett. 86, 1370 (2001).

21 Yin, J., et al. Quantum teleportation and entanglement distribution over 100-kilometre free-space channels. Nature 488, 185 (2012).

22 Ma, X.-S., et al. Quantum teleportation over 143 kilometres using active feed-forward. Nature 489, 269 (2012).

23 Marcikic, I., de Riedmatten, H., Tittel, W., Zbinden, H. \& Gisin, $\mathrm{N}$. Long-distance teleportation of qubits at telecommunication wavelengths. Nature 421, 509 (2003).

${ }^{24}$ de Riedmatten, H., et al. Long distance quantum teleportation in a quantum relay configuration. Phys. Rev. Lett. 92, 047904 (2004).

25 Landry, O., et al. Quantum teleportation over the Swisscom telecommunication network. J. Opt. Soc. Am. B 24, 398-403, (2007).

${ }^{26}$ Metcalf, B. J., et al. Quantum teleportation on a photonic chip. Nature Photonics 8, 770-774 (2014).

27 Wang, X.-L. Quantum teleportation of multiple degrees of freedom in a single photon. Nature 518, 516-519 (2015).

${ }^{28}$ Nielsen, M. A., Knill, E. \& Laflamme, R. Complete quantum teleportation using nuclear magnetic resonance. Nature 396, 52 (1998).

29 Furusawa, A., et al. Unconditional quantum teleportation. Science 282, 706 (1998).

${ }^{30}$ Bowen, W. P., et al. Experimental investigation of continuousvariable quantum teleportation. Phys. Rev. A 67, 032302 (2003).

31 Zhang, T. C., Goh, K. W., Chou, C. W., Lodahl, P \& Kimble, H. J. Quantum teleportation of light beams. Phys. Rev. A 67, 033802 (2003).

32 Takei, N., Yonezawa, H., Aoki, T. \& Furusawa, A. Highfidelity teleportation beyond the no-cloning limit and entangle- 
ment swapping for continuous variables. Phys. Rev. Lett. 94, 220502 (2005).

33 Yonezawa, H., Braunstein, S. L. \& Furusawa, A. Experimental demonstration of quantum teleportation of broadband squeezing. Phys. Rev. Lett. 99, 110503 (2007).

34 Takei, N., et al. Experimental demonstration of quantum teleportation of a squeezed state. Phys. Rev. A 72, 042304 (2005).

35 Lee, N., et al. Teleportation of nonclassical wave packets of light. Science 332, 330-333 (2011).

36 Yukawa, M., Benichi, H. \& Furusawa, A. High-fidelity continuous-variable quantum teleportation toward multistep quantum operations. Phys. Rev. A 77, 022314 (2008).

37 Takeda, S., Mizuta, T., Fuwa, M., van Loock, P. \& Furusawa, A. Deterministic quantum teleportation of photonic quantum bits by a hybrid technique. Nature 500, 315 (2013).

38 Sherson, J. F., et al. Quantum teleportation between light and matter. Nature 443, 557 (2006).

39 Krauter, H., et al. Deterministic quantum teleportation between distant atomic objects. Nature Phys. 9, 400 (2013).

${ }^{40}$ Chen, Y.-A., et al. Memory-built-in quantum teleportation with photonic and atomic qubits. Nature Phys. 4, 103 (2008).

41 Bao, X-H., et al. Quantum teleportation between remote atomic ensemble quantum memories. Proc. Natl Acad. Sci. USA 109, 20347-20351 (2012).

${ }^{42}$ Barrett, M. D., et al. Deterministic quantum teleportation of atomic qubits. Nature 429, 737 (2004).

43 Riebe, M., et al. Deterministic quantum teleportation with atoms. Nature 429, 734 (2004).

44 Riebe, M., et al. Quantum teleportation with atoms: quantum process tomography. New J. Phys. 9, 211 (2007).

45 Olmschenk, S., et al. Quantum teleportation between distant matter qubits. Science 323, 486 (2009).

46 Nölleke, C., et al. Efficient teleportation between remote singleatom quantum memories. Phys. Rev. Lett. 110, 140403 (2013).

47 Gao, W. B., et al. Quantum teleportation from a propagating photon to a solid-state spin qubit. Nature Comm. 4, 1 (2013).

48 Bussières, F., et al. Quantum teleportation from a telecomwavelength photon to a solid-state quantum memory. Nature Photonics 8, 775-778 (2014).

49 L. Steffen, L., et al. Deterministic quantum teleportation with feed-forward in a solid state system. Nature 500, 319 (2013).

${ }^{50}$ Pfaff, W., et al. Unconditional quantum teleportation between distant solid-state quantum bits. Science 345, 532 (2014).

51 Weinfurter, H. Experimental Bell-state analysis. Europhys. Lett. 25, 559-564 (1994).

52 Braunstein, S. L. \& Mann, A. Measurement of the Bell operator and quantum teleportation. Phys. Rev. A 51, R1727-R1730 (1995).

53 Calsamiglia, J. \& Lütkenhaus, N. Maximum efficiency of a linear-optical Bell-state analyzer. Appl. Phys. B 72, 67 (2001).

54 Bennett, C. H., et al. Remote state preparation. Phys. Rev. Lett. 87, 077902 (2001).

55 Scarani, V., Iblisdir, S., Gisin, N. \& Acín, A. Quantum cloning. Rev. Mod. Phys. 77, 1225 (2005).

56 Massar, S. \& Popescu, S. Optimal extraction of information from finite quantum ensembles. Phys. Rev. Lett. 74, 1259-1263 (1995).

57 Werner, R. F. All teleportation and dense coding schemes. $J$. Phys. A 34, 7081 (2001).

58 Vaidman, L. Teleportation of quantum states. Phys. Rev. A 49, 1473 (1994).

59 Braunstein, S. L. \& Kimble, H. J. Teleportation of continuous quantum variables. Phys. Rev. Lett. 80, 869 (1998).

60 Pirandola, S., Mancini, S., Vitali, D. \& Tombesi, P. Continuous variable entanglement and quantum state teleportation be- tween optical and macroscopic vibrational modes through radiation pressure. Phys. Rev. A 68, 062317 (2003).

${ }^{61}$ Mancini, S., Vitali, D. \& Tombesi, P. Scheme for teleportation of quantum states onto a mechanical resonator. Phys. Rev. Lett. 90, 137901 (2003).

${ }^{62}$ Kuzmich, A. \& Polzik, E. S. Atomic quantum state teleportation and swapping. Phys. Rev. Lett. 85, 5639 (2000).

63 Duan, L.-M., Cirac, J. I., Zoller, P. \& Polzik, E. S. Quantum communication between atomic ensembles using coherent light. Phys. Rev. Lett. 85, 5643 (2000).

${ }^{64}$ Hammerer, K., Srensen, A. \& Polzik, E.S. Quantum interface between light and atomic ensembles. Rev. Mod. Phys. 82, 10411093 (2010).

65 Eisert, J. Entanglement in quantum information theory. $\mathrm{PhD}$ thesis (Potsdam, February 2001).

66 Vidal, G. \& Werner, R. F. Computable measure of entanglement. Phys. Rev. A 65, 032314 (2002).

${ }^{67}$ Plenio, M. B. Logarithmic negativity: A full entanglement monotone that is not convex. Phys. Rev. Lett. 95, 090503 (2005).

${ }^{68}$ Braunstein, S. L., Fuchs, C. A., Kimble, H. J. \& van Loock, P. Quantum versus classical domains for teleportation with continuous variables. Phys. Rev. A 64, 022321 (2001).

69 Hammerer, K., Wolf, M. M., Polzik, E. S. \& Cirac, J. I. Quantum benchmark for storage and transmission of coherent states. Phys. Rev. Lett. 94, 150503 (2005).

${ }^{70}$ Grosshans, F. \& Grangier, P. Quantum cloning and teleportation criteria for continuous quantum variables. Phys. Rev. A 64, 010301(R) (2001).

71 Pirandola, S. \& Mancini, S. Quantum teleportation with continuous variables: A survey. Laser Physics 16, 1418 (2006).

72 Fiurásěk, J., Improving the fidelity of continuous-variable teleportation via local operations. Phys. Rev. A 66, 012304 (2002).

73 Pirandola, S., Mancini S. \& Vitali, D. Conditioning two-party quantum teleportation within a three-party quantum channel. Phys. Rev. A 71, 042326 (2005).

74 Pirandola, S. A Quantum Teleportation Game. Int. J. Quant. Inf. 3, 239 (2005).

75 Adesso, G. \& Chiribella G. Quantum Benchmark for Teleportation and Storage of Squeezed States. Phys. Rev. Lett. 100, 170503 (2008).

76 Chiribella, G. \& Adesso, G. Quantum benchmarks for pure single-mode Gaussian states. Phys. Rev. Lett. 112, 010501 (2014).

77 Braunstein, S. L., D’Ariano, G. M., Milburn, G. J. \& Sacchi, M. F. Universal teleportation with a twist. Phys. Rev. Lett. 84, 3486 (2000).

78 Abramsky, S. \& Coecke, B. A categorical semantics for quantum protocols. Proc. of LICS'04, IEEE Computer Science Press (2004).

79 van Loock, P., Braunstein, S. L. \& Kimble, H. J. Broadband teleportation. Phys. Rev. A 62, 022309 (2000).

80 Zukowski, M., Zeilinger, A., Horne, M. A. \& Ekert, A. "Eventready-detectors" Bell experiment via entanglement swapping. Phys. Rev. Lett. 71, 4287 (1993).

81 van Loock, P. \& S. L. Braunstein. Unconditional teleportation of continuous-variable entanglement. Phys. Rev. A 61, 010302(R) (1999).

82 Polkinghorne, R. E. S. \& T. C. Ralph. Continuous variable entanglement swapping. Phys. Rev. Lett. 83, 2095 (1999).

83 Tan, S. M. Confirming entanglement in continuous variable quantum teleportation. Phys. Rev. A 60, 2752 (1999).

84 Johnson, T. J., Bartlett, S. D. \& Sanders, B. C. Continuousvariable quantum teleportation of entanglement. Phys. Rev. A 66, $042326(2002)$ 
85 Pirandola, S., Vitali, D., Tombesi, P. \& Lloyd, S. Macroscopic Entanglement by entanglement swapping. Phys. Rev. Lett. 97, 150403 (2006).

${ }^{86}$ Abdi, M., Pirandola, S., Tombesi, P. \& Vitali, D. Entanglement swapping with local certification: Application to remote micromechanical resonators. Phys. Rev. Lett. 109, 143601 (2012).

${ }^{87}$ Pan, J.-W., Bouwmeester, D., Weinfurter, H. \& Zeilinger, A. Experimental entanglement swapping: Entangling photons that never interacted. Phys. Rev. Lett. 80, 3891 (1998).

${ }^{88}$ Pan, J.-W., Daniell, M., Gasparoni, S., Weihs, G. \& Zeilinger, A. Experimental demonstration of four-photon entanglement and high-fidelity teleportation. Phys. Rev. Lett. 86, 435 (2001).

89 Jennewein, T., Weihs, G., Pan, J.-W. \& Zeilinger, A. Experimental Nonlocality Proof of quantum teleportation and entanglement swapping. Phys. Rev. Lett. 88, 017903 (2002).

$90 \mathrm{Jia}, \mathrm{X}$., et al. Experimental demonstration of unconditional entanglement swapping for continuous variables. Phys. Rev. Lett. 93, 250503 (2004).

91 Takeda, S., Fuwa, M., van Loock, P. \& Furusawa, A. Entanglement swapping between discrete and continuous variables. Phys. Rev. Lett. 114, 100501 (2015).

92 Bennett, C. H., et al. Purification of noisy entanglement and faithful teleportation via noisy channels. Phys. Rev. Lett. 76, 722 (1996).

93 Eisert, J., Browne, D. E., Scheel, S. \& Plenio, M. B. Distillation of continuous-variable entanglement with optical means. Annals of Physics 311, 431-458 (2004).

94 Braunstein, S. L. \& Pirandola, S. Side-channel-free quantum key distribution. Phys. Rev. Lett. 108, 130502 (2012).

95 Karlsson, A. \& Bourennane, M. Quantum teleportation using three-particle entanglement. Phys. Rev. A 58, 4394 (1998).

96 Hillery, M., Buzek, V. \& Berthiaume, A. Quantum secret sharing. Phys. Rev. A 59, 1829 (1999).

97 van Loock, P. \& Braunstein, S. L. Multipartite entanglement for continuous variables: A quantum teleportation network. Phys. Rev. Lett. 84, 3482 (2000).

98 Yonezawa, H., Aoki, T. \& Furusawa, A. Demonstration of a quantum teleportation network for continuous variables. Nature 431, 430 (2004).

99 Lance, A. M., Symul, T., Bowen, W. P., Sanders, B. C. \& Lam, P. K. Tripartite quantum state sharing. Phys. Rev. Lett. 92, 177903 (2004).

100 Bužek, V. \& Hillery, M. Quantum copying: Beyond the nocloning theorem. Phys. Rev. A 54, 1844 (1996).

101 Bruß, D., et al. Optimal universal and state-dependent quantum cloning. Phys. Rev. A 57, 2368 (1998).

102 Cerf, N. J., Ipe, A. \& Rottenberg, X. Cloning of continuous quantum variables. Phys. Rev. Lett. 85, 1754 (2000).

103 Zhao, Z., et al. Experimental realisation of optimal asymmetric cloning and tele-cloning via partial teleportation. Phys. Rev. Lett. 95, 030502 (2005).

104 Koike, S., et al. Demonstration of quantum tele-cloning of Optical Coherent States. Phys. Rev. Lett. 96, 060504 (2006).

105 Murao, M., Jonathan, D., Plenio, M. B. \& Vedral, V. Quantum tele-cloning and multiparticle entanglement. Phys. Rev. A 59, 156 (1999).

106 van Loock, P. \& Braunstein, S. L. Telecloning of continuous quantum variables. Phys. Rev. Lett. 87, 247901 (2001).

107 Brassard, G., Braunstein, S. L. \& Cleve, R. Teleportation as a quantum computation. Physica D 120, 43-47 (1998).

108 Aliferis, P. \& Leung, D. W. Computation by measurements: A unifying picture, Phys. Rev. Lett. 70, 062314 (2004).

109 Knill, E., Laflamme, R. \& Milburn, G. A scheme for efficient quantum computation with linear optics. Nature 409, 46-52
(2001).

110 Gao, W.-B., et al. Teleportation-based realisation of an optical quantum two-qubit entangling gate. Proceedings of the National Academy of Sciences 107, 20869-20874 (2010).

111 Gross, D. \& Eisert, J. Novel schemes for measurement-based quantum computing, Phys. Rev. Lett. 98, 220503 (2007).

112 Nielsen, M. A. Optical quantum computation using cluster states. Phys. Rev. Lett. 93, 040503 (2004).

113 Menicucci, N. C., et al. Universal quantum computation with continuous-variable cluster states. Phys. Rev. Lett. 97, 110501 (2006).

114 Zhang, J. \& Braunstein, S. L. Continuous-variable Gaussian analog of cluster states. Phys. Rev. A 73, 032318 (2006).

115 Yokoyama, S., et al. Ultra-large-scale continuous-variable cluster states multiplexed in the time domain. Nature Photonics 7, 982986 (2013).

116 Ishizaka, S. \& Hiroshima, T. Quantum teleportation scheme by selecting one of multiple output ports. Phys. Rev. A 79, 042306 (2009).

117 Strelchuk, S., Horodecki, M., \& Oppenheim, J. Generalised teleportation and entanglement recycling. Phys. Rev. Lett. 110, 010505 (2013).

118 Beigi, S. \& König, R. Simplified instantaneous non-local quantum computation with applications to position-based cryptography. New J. Phys. 13, 093036 (2011).

119 Buhrman, H., et al. Quantum communication complexity advantage implies violation of a Bell inequality, Preprint arXiv:1502.0105v1 (2015).

${ }^{120}$ Grice, W. P. Arbitrarily complete Bell-state measurement using only linear optical elements. Phys. Rev. A 84, 042331 (2011).

121 Zaidi, H. A. \& van Loock, P. Beating the One-Half Limit of Ancilla-Free Linear Optics Bell Measurements. Phys. Rev. Lett. 110, 260501 (2013).

122 Ewert, F. \& van Loock, P. 3/4-Efficient Bell Measurement with Passive Linear Optics and Unentangled Ancillae. Phys. Rev. Lett. 113, 140403 (2014).

123 Braunstein, S. L., \& Kimble H. J., A posteriori teleportation. $\mathrm{Na}$ ture 394, 840 (1998).

124 Brendel, J., Tittel, W., Zbinden, H. \& Gisin, N. Pulsed energytime entangled twin-photon source for quantum communication. Phys. Rev. Lett. 82, 2594-2597 (1999).

125 Furusawa, A. \& van Loock, P. Quantum teleportation and entanglement -A hybrid approach to optical quantum information processing (Wiley-VCH, Weinheim, 2011).

126 Andersen, U. L. \& Ralph, T. C. High-fidelity teleportation of continuous-variable quantum states using delocalised single photons. Phys. Rev. Lett. 111, 050504 (2013).

127 Lvovsky, A. I., Sanders, B. C. \& Tittel, W. Optical quantum memory. Nature Photonics 3, 706-714 (2009).

128 Simon, C., et al. Quantum memories. A review based on the European Integrated Project "Qubit Applications (QAP)". Eur. Phys. J. D 58, 1 (2010).

129 Sangouard, N., Simon, C., de Riedmatten, H. \& Gisin, N. Quantum repeaters based on atomic ensembles and linear optics. Rev. Mod. Phys. 83, 33 (2011).

130 Bussires, F., et al. Prospective applications of optical quantum memories. J. of Mod. Opt. 60, 1519-1537 (2013).

131 Julsgaard, B., et al. Experimental demonstration of quantum memory for light. Nature 432, 482 (2004).

132 Radnaev, A. G., et al. A quantum memory with telecomwavelength conversion. Nat. Phys. 6, 894899 (2010).

133 Zhong, M. et al. Optically addressable nuclear spins in a solid with a six-hour coherence time. Nature 517, 177 (2015). 
${ }^{134}$ Maurer, P. C., et al., Room-Temperature Quantum Bit Memory Exceeding One Second. Science 336, 1283-1286 (2012).

135 Harty, T. P., et al. High-fidelity preparation, gates, memory, and readout of a trapped-ion quantum bit. Phys. Rev. Lett. 113, 220501 (2014).

136 Specht, H. P., et al. A single-atom quantum memory. Nature 473, 190 (2011).

137 Press, D., et al. Ultrafast optical spin echo in a single quantum dot. Nat. Photonics 4, 367 (2010).

138 Jobez, P., et al. Coherent spin control at the quantum level in an ensemble-based optical memory. Preprint arXiv:1501.03981.
139 Xiang, Z.-L., Ashhab, S., You, J. Q. \& Nori, F. Hybrid quantum circuits: Superconducting circuits interacting with other quantum systems. Rev. Mod. Phys. 85, 623-653 (2013).

${ }^{140}$ Kurizki, G., Bertet, P., Kubo, Y., Mølmer, K., Petrosyan, D., Rabl, P. \& Schmiedmayer, J. Quantum technologies with hybrid systems. Proc. Natl. Acad. Sci. USA 112, 3866-73 (2015).

141 Bar-Gill, N., Pham, L. M., Jarmola, A., Budker, D. \& Walsworth, R. L. Solid-state electronic spin coherence time approaching one second. Nat. Commun. 4, 1743 (2013).

142 Schoelkopf, R. J. \& Girvin, S. M. Wiring up quantum systems. Nature 451, 664669 (2008).

143 O'Brien, C., Lauk, N., Blum, S., Morigi, G. \& Fleischhauer, M. Interfacing Superconducting Qubits and Telecom Photons via a Rare-Earth-Doped Crystal. Phys. Rev. Lett. 113, 063603 (2014). 